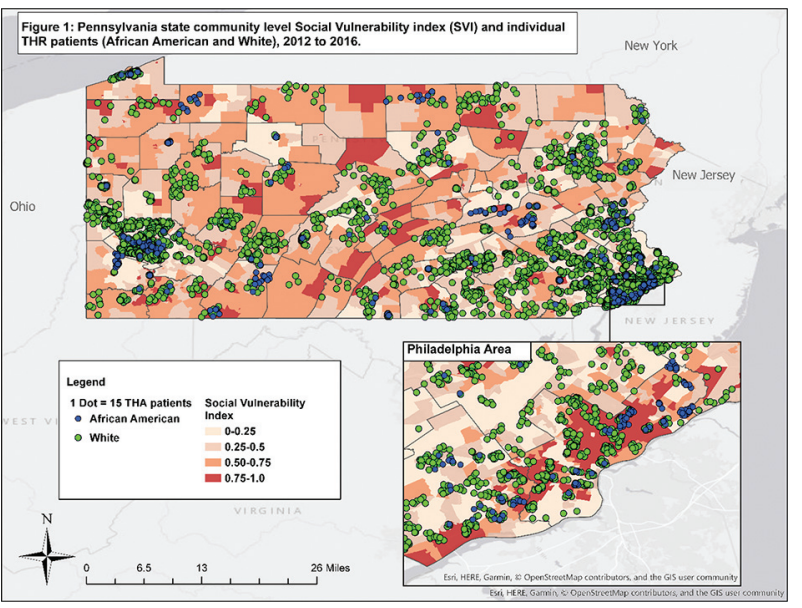

Abstract THU0442 - Figure 1. Pennsylvania state community level Social Vulnerability index (SVI) and individual Total Hip Replacement patients (African American and White), 2012 to 2016

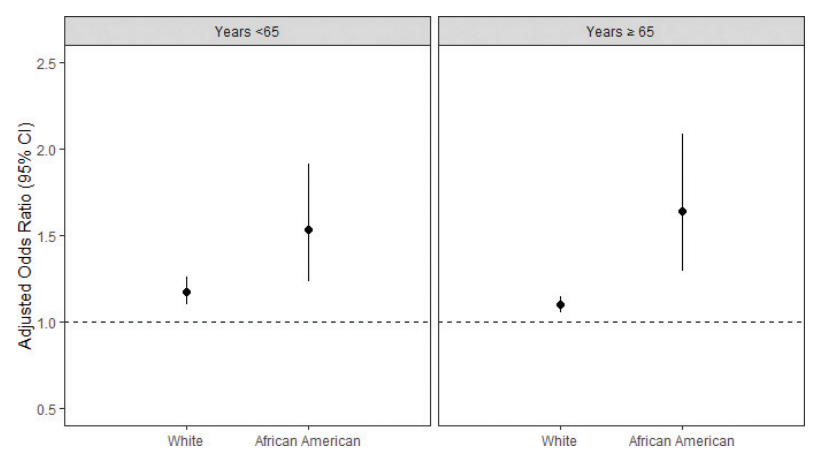

Abstract THU0442 - Figure 2. Adjusted odds of discharge to an Institution (vs Home) for patients living in High SVI compared to Low SVI stratified by race

${ }^{a}$ Adjusted covariates for all models include patient sex, age, insurance, facility metro status, volume of cases, surgical complications, and Elixhauser Index.

Disclosure of Interests: Bella Mehta: None declared, Susan Goodman Grant/research support from: Novartis: research support, Consultant for: Novartis, UCB, Pfizer: consulting, Kaylee Ho: None declared, Debra D'Angelo: None declared, Michael Parks Grant/research support from: Zimmer Biomet, Employee of: Zimmer Biomet, Said Ibrahim: None declared DOI: 10.1136/annrheumdis-2019-eular.6114

\section{THU0443 ASSESSING PAIN CHARACTERISTICS IN PERSONS WITH HAND OSTEOARTHRITIS USING THE MCGILL PAIN QUESTIONNAIRE}

Elisabeth Mulrooney ${ }^{1}$, Karin Magnusson ${ }^{2,3}$, Hilde Berner Hammer ${ }^{1}$, Hanne Solveig Dagfinrud ${ }^{2,4}$, Tore K. Kvien ${ }^{1,5}$, Ida Kristin Haugen ${ }^{1} .{ }^{1}$ Diakonhjemmet Hospital, Department of Rheumatology, Oslo, Norway, ${ }^{2}$ Diakonhjemmet Hospital, National Advisory Unit on Rehabilitation in Rheumatology, Oslo, Norway; ${ }^{3}$ Lund University, Department of Orthopedics, Lund, Sweden; ${ }^{4}$ University of Oslo, Department of Interdiciplinary Health Sciences, Oslo, Norway; ${ }^{5}$ University of Oslo, Department of Orthopaedics, Oslo, Norway

Background: Pain in osteoarthritis (OA) causes significant physical, psychological and social limitations. Increased understanding of the pain experience is important for improved patient care.

Objectives: We aimed to assess the pain characteristics of hand OA using the McGill Pain Questionnaire, a multidimensional self-reporting questionnaire that assesses the quality and intensity of the pain. Secondly, the external validity was examined.

Methods: Three-hundred persons with hand OA from the Nor-Hand study completed questionnaires, including a modified McGill questionnaire. The participants are presented with a list of 106 pain descriptors in 18 sections. The questionnaire consists of three subscales: sensory (12 sections, $0-71.3$ scale), affective ( 5 sections, 0-32.9 scale) and evaluative (one section, 0-8.6 scale), and an intensity score (no pain - severe pain). The total scale is rated from $0-112.8$. The subscales and total scale were normalized to $0-100$ scales. The participants also completed the Numeric Rating Scale (NRS, 0-10 scale) about hand pain, the Australian/ Canadian (AUSCAN) hand pain subscale (0-20 scale) and questionnaires about psychological factors; Hospital Depression and Anxiety Scale (HADS, 0-42 scale), Pain Catastrophizing Scale (PCS, 0-52 scale) and the pain subscale of the Arthritis Self Efficacy Scale (ASES, 10-100 scale). For all scales except ASES, high scores indicate poor health. We identified the adjectives in the McGill questionnaire with most frequent responses. To determine the external validity, the McGill questionnaire was correlated to other questionnaires (AUSCAN, NRS, HADS, PCS and ASES) using Spearman correlation coefficients.

Results: The participants (89\% women) with median (IQR) age of 61 (57-66) years demonstrated a wide range in pain characteristics and intensity, with a median (IQR) of $34.5(0-62.3), 14.9(0-39.2)$ and 44.2 (34.9-48.8) for the sensory, affective and evaluate subscales, respectively. The median for the total pain sum score was 29.7 (7.0-53.2). A floor effect was detected for the sensory $(23.7 \%$ with score 0$)$ and affective (40.3\% with score 0$)$ subscales. The reported adjectives with highest frequency were "sore" ( $n=208,69.3 \%)$, "inhibiting" ( $n=124,41.3 \%)$ and "annoying" ( $n=97,32.3 \%)$. The participants frequently reported neuropathiclike characteristics such as sticking/stabbing/pricking $(n=137,45.7 \%)$, speeding/radiant $(n=110,36,6 \%), \quad$ smarting/burning $(n=89,29.6 \%)$ and creeping ( $n=43,14.3 \%)$.

The McGill total scale showed moderate correlations with AUSCAN and NRS pain (Table). Similar strength of correlations was found for all the subscales, and stronger for the intensity score (0.63-0.64). The correlation with HADS was similar for the McGill questionnaire, AUSCAN and NRS pain. The correlations with PCS and ASES were weaker for McGill than for the other pain questionnaires.

Abstract THU0443 -Table 1. Correlations between the McGill questionnaire and questionnaires assessing pain and psychological health.

\begin{tabular}{lcccccc}
\hline & $\begin{array}{c}\text { McGill } \\
\text { total }\end{array}$ & AUSCAN & $\begin{array}{c}\text { NRS } \\
\text { pain }\end{array}$ & HADS & PCS & ASES \\
\hline McGill & 1.0 & & & & & \\
total & & 1.0 & & & & \\
AUSCAN & 0.41 & 0.73 & 1.0 & & & \\
NRS pain & 0.45 & 0.24 & 0.29 & 1.0 & & \\
HADS & 0.31 & 0.40 & 0.41 & 0.47 & 1.0 & \\
PCS & 0.26 & -0.35 & -0.40 & -0.30 & -0.42 & 1.0 \\
ASES & -0.20 & & & & &
\end{tabular}

Conclusion: The McGill Pain questionnaire may be a useful tool in research settings for a broad evaluation of pain characteristics in hand OA. Moderate correlations with other pain questionnaires suggest that the McGill questionnaire measures other constructs. For the first time, we have shown that neuropathic-like pain characteristics are frequently reported by persons with hand OA.

Disclosure of Interests: Elisabeth Mulrooney: None declared, Karin Magnusson: None declared, Hilde Berner Hammer Grant/research support from: AbbVie, Pfizer and Roche, Paid instructor for: AbbVie, Pfizer, UCB, Novartis, Roche, Speakers bureau: AbbVie, Pfizer, UCB, Novartis, Roche, Hanne Solveig Dagfinrud Consultant for: Honoraria from Novartis as a steering committee member on this survey, Tore K. Kvien Grant/research support from: AbbVie, BMS, MSD, Pfizer, Roche and UCB., Consultant for: AbbVie, Biogen, BMS, Boehringer Ingelheim, Celgene, Celltrion, El Lilly, Hospira, Merck-Serono, MSD, Novartis, Oktal, Orion Pharma, Pfizer, Roche, Sandoz, Sanofi, Mylan and UCB, Speakers bureau: AbbVie, Biogen, BMS, Boehringer Ingelheim, Celgene, Celltrion, Eli Lilly, Hospira, Merck-Serono, MSD, Novartis, Oktal, Orion Pharma, Pfizer, Roche, Sandoz, Sanofi and UCB, Ida Kristin Haugen Grant/research support from: ADVANCE research grant from Pfizer, Consultant for: Advisory board Abbvie

DOI: 10.1136/annrheumdis-2019-eular.4606

\section{THU0444 CROSS-CULTURAL TRANSLATION, ADAPTATION AND VALIDATION OF A JAPANESE VERSION OF THE FUNCTIONAL INDEX FOR HAND OSTEOARTHRITIS (J- FIHOA)}

Yasunobu Nakagawa ${ }^{1}$, Shigeru Kurimoto ${ }^{1}$, Emmanuel Maheu ${ }^{2}$, Renée Liliane Dreiser ${ }^{3}$, Hitoshi Hirata $1 .{ }^{1}$ Nagoya University Hospital, Hand Surgery, Nagoya, Japan; ${ }^{2}$ Hôpital Saint-Antoine, APHP, Rheumatology, Paris, France; ${ }^{3}$ Hopital Bichât, APHP, Rheumatology, Paris, France

Background: Hand osteoarthritis (HOA) is a highly prevalent and heterogeneous musculoskeletal disorder. Although well-designed clinical trials and guidelines have been published, there are comparatively few HOA 
studies concerning Asian countries. To both pursue appropriate clinical courses for patients and to engage in future research, physicians need to discriminate symptomatic HOA. The Functional Index for Hand OsteoArthritis (FIHOA) assesses hand OA-related functional impairment and is accepted as a gold standard with excellent reliability and responsiveness $[1,2]$.

Objectives: Our objective was to make a Japanese version of the FIHOA (J-FIHOA) and to validate it among Japanese HOA patients.

Methods: J-FIHOA was created following forward/backward translation processes distilled from the established guidelines. A prospective, multicenter study was undertaken for its validation. Seventeen collaborating hospitals recruited hand OA patients from September 2017 to September 2018. Patients who met the ACR classification criteria were included. A medical record review and the following patient-rated questionnaires were collected: J-FIHOA, Japanese Health Assessment Questionnaire (J-HAQ), numerical rating scale for pain (NRS pain) and Japanese Short Form 36 Health Survey (J-SF-36). As item 7 is a gender-role question, an item total correlation needed to be calculated independently for women and men.The J-FIHOA scores at enrollment were used to assess Cronbach's alpha coefficient/item-total correlation and compared with the J-HAQ, NRS pain and J-SF-36 yielding respective Spearman's rank correlation coefficients. To assess reliability, we used test-retest methods for patients with unchanged symptoms/treatments. The interval was 1-2 weeks and data sets were assessed with the intraclass correlation coefficient (ICC). To evaluate responsiveness, those who started new pharmacological treatments were required to answer the questionnaires and to have a 1month follow-up visit. Differences between pre- and post- pharmacological treatments were used to calculate the effect size (ES)

Results: Twenty-nine male and 145 female HOA patients participated (mean age 65 years). Cronbach's alpha was 0.91 , showing high internal consistency. Each item was well correlated with the total score (all values $>0.30$; range 0.46 to 0.89 ). Construct validity between J-FIHOA and other scales was: 0.73 (J-HAQ), 0.56 (NRS pain), -0.31 (J-SF-36 physical component) and -0.21 (J-SF-36 mental component). Although the FIHOA has no pain-related question, previous reports showed moderate correlations between the FIHOA and pain. Our results were concordant with these reports. It was expected that J-SF-36 mental component had the weakest correlation. One hundred fifty-seven longitudinal data sets were pooled and used to evaluate reliability (137 for test-retest) and responsiveness (20 for ES). ICC for test-retest was $0.83(95 \% \mathrm{Cl}, 0.77$ to 0.88). The ES were 0.56 for J-FIHOA and 0.44 for J-HAQ. We assumed that this higher ES related on the HOA specificity of J-FIHOA.

Conclusion: We created a cross-culturally adapted J-FIHOA. Our results showed its good metric qualities to assess dysfunction in HOA and equivalence with the original FIHOA.

\section{REFERENCES:}

[1] Dreiser RL, Maheu E, et al. Validation of an algofunctional index for osteoarthritis of the hand. Revue du rhumatisme (English ed). 1995; 62(6 Suppl 1):43s-53s.

[2] Dreiser RL, Maheu E, et al. Sensitivity to change of the functional index for hand osteoarthritis. Osteoarthritis Cartilage. 2000; 8:S25-S28.

Disclosure of Interests: None declared

DOI: 10.1136/annrheumdis-2019-eular.2025

\section{THU0445 ASSOCIATION BETWEEN PATIENT'S EXPECTATION AND SATISFACTION FOLLOWING TOTAL KNEE REPLACEMENT FOR OSTEOARTHRITIS}

Maria Noviani ${ }^{1}$, Julian Thumboo ${ }^{1,2}$, Seng Jin $Y^{2} o^{3}$, Steven Bak-Siew Wong ${ }^{4}$, Vikki Wylde ${ }^{5}$, Bibhas Chakraborty ${ }^{2}$, Ngai Nung Lo ${ }^{3}$, William Yeo ${ }^{3}$, Hwei Chi Chong ${ }^{3}$, Mann Hong Tan ${ }^{3}$, Hee Nee Pang ${ }^{3}$, Darren Tay ${ }^{3}$, Ying Ying Leung ${ }^{1,2}$.

${ }^{1}$ Singapore General Hospital, Department of Rheumatology and Immunology, Singapore, Singapore; ${ }^{2}$ Duke-NUS Medical School, Singapore, Singapore; ${ }^{3}$ Singapore General Hospital, Department of Orthopaedic Surgery, Singapore, Singapore; ${ }^{4}$ Singapore General Hospital, Department of Radiology, Singapore, Singapore; ${ }^{5}$ University of Bristol, Musculoskeletal Research Unit, Translational Health Sciences, Bristol, United Kingdom

Background: Up to $20 \%$ of patients are dissatisfied with the outcome of total knee replacement (TKR). Pre-operative expectations may affect patient's outcomes, including satisfaction after surgery. Better understanding of patient's expectation could aid the development and improvements of pre-operative education. Pre-operative expectations and post-operative satisfaction may vary across cultures and have not been described in an Asian context.
Objectives: We aimed to evaluate the association between patient's preoperative expectation and post-operative satisfaction following TKR for osteoarthritis.

Methods: Patients listed for TKR because of $O A$ in a tertiary referral centre were recruited. Pre-operatively, participants completed sociodemographic questions, Western Ontario and McMaster Universities Index (WOMAC) pain and function, and the Hospital for Special Surgery (HSS) $\mathrm{KR}$ expectation survey. At 6 months after TKR, patients rated their satisfaction with each expectation on the HSS as completely satisfied, partially satisfied or dissatisfied. Overall satisfaction with TKR was assessed with a 4-point Likert scale and dichotomized to satisfied or dissatisfied. Association between overall satisfaction at 6 months and pre-operative expectation was analysed using logistic regression after adjustment for demographic data.

Results: Between June 2017 and December 2017, 215 patients were recruited and completed follow up at 6 months after TKR $(71 \%$ female, $82 \%$ Chinese, mean age $65 \pm 7$ years). The overall satisfaction rate was $92 \%$. The most prevalent pre-operative expectations were improved ability to perform daily activities $(85 \%)$, participation in recreation $(83 \%)$, pain relief $(81 \%)$ and ability to change position $(81 \%)$ (Figure 1 ). At 6 month after TKR, the top five fulfilled expectations (defined as 'completely satis fied') were improvement in the ability to perform daily activities (82\%), walking short distance $(66 \%)$, improvement in psychological well-being $(64 \%)$, pain relief $(64 \%)$ and position change (56\%) (Figure 1). For most items on the questionnaire, expectations were high and not fully met; however most patient's expectations were at least partially met. In the logistic regression model, post-operative HSS total score, but not preoperative HSS score, was associated with overall satisfaction $(P<0.01)$ after adjusting for demographic data (Table 1).

Abstract THU0445 - Table 1. Variables Associated with Overall Satisfaction at 6 Months after TKR by Logistic Regression Analysis

\begin{tabular}{lcc}
\hline & $\begin{array}{c}\text { Exp(B), (95\% Confidence } \\
\text { Interval) }\end{array}$ & P \\
\hline Age & $0.985(0.869,1.115)$ & 0.809 \\
Gender & $0.589(0.128,2.700)$ & 0.496 \\
Ethnic group & $0.729(0.093,5.741)$ & 0.764 \\
Education level & $0.142(0.010,2.065)$ & 0.153 \\
BMI & $0.992(0.872,1.128)$ & 0.901 \\
Radiographic index of knee & $0.917(0.228,3.689)$ & 0.902 \\
WOMAC pain, baseline & $0.988(0.926,1.053)$ & 0.710 \\
WOMAC function, baseline & $0.978(0.903,1.060)$ & 0.589 \\
HSS total score at baseline & $1.024(0.967,1.084)$ & 0.421 \\
HSS total score at 6 & $1.172(1.092,1.259)$ & $<0.001$ \\
months & & \\
\hline
\end{tabular}

Conclusion: Although most patient's expectations were at least partially met, they were not fully met. This suggests that many patients have unrealistic expectations of TKR outcome. Fulfilment of expectations, rather than expectation per se, was the most important variable associated with patient's overall satisfaction.

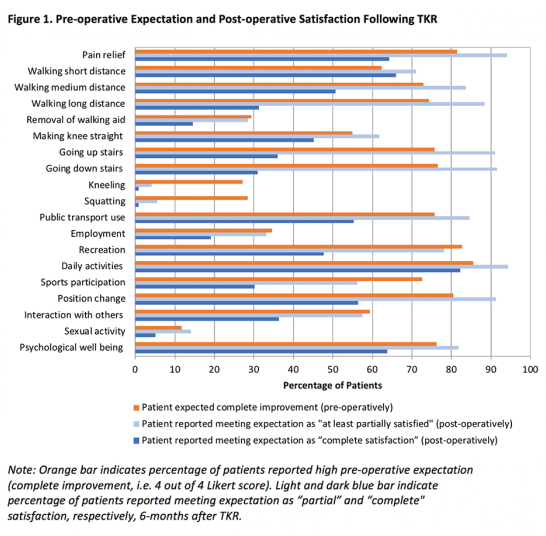

Abstract THU0445 - Figure 1

Disclosure of Interests: Maria Noviani: None declared, Julian Thumboo: None declared, Seng Jin Yeo: None declared, Steven Bak-Siew Wong: None declared, Vikki Wylde: None declared, Bibhas Chakraborty: None declared, Ngai Nung Lo: None declared, William Yeo: None declared Hwei Chi Chong: None declared, Mann Hong Tan: None declared, Hee 